\title{
Performance analysis and power allocation strategy in single-antenna cognitive overlay systems
}

\author{
Wei-Lun Lin ${ }^{1 *}$ (D), Chih-Hao Lin² and Fan-Shuo Tseng ${ }^{2}$
}

\begin{abstract}
A power allocation strategy is proposed for single-antenna overlay cognitive radio networks, in which the secondary user helps transmit the signal of the primary user while concurrently conveying its own signal by means of a superposition coding technique. The study commences by deriving analytical expressions for the bit error rates (BERs) of the primary and secondary users. A power allocation strategy is then proposed for minimizing the total power consumption of the two users while simultaneously satisfying their respective BER constraints. The analytical BER formulas are not convex, and hence the optimization process presents a significant challenge. Accordingly, two more tractable BER approximations for the primary and secondary users are proposed to transfer the non-convex problem into a convex one. The simulation results confirm the effectiveness of the proposed power allocation strategy under various channel environments.
\end{abstract}

Keywords: Cognitive radio; Overlay design; Cooperative relaying; Superposition coding; Power allocation

\section{Introduction}

Cognitive radio (CR) transceiver design has received significant attention in recent years due to the ability it provides to make more efficient use of the spectrum resources [1-3]. By assigning the CR users different priorities, i.e., primary users (or non-cognitive users), denoted as $U_{A}$, and secondary users (or cognitive users), denoted as $U_{B}$, both types of user are able to coexist in a neat way and fully utilize all of the spectrum resources. As a result, CR provides a highly promising solution for future wireless systems [4].

In developing CR networks, the aim is for $U_{B}$ to maximize its use of the spectrum resources without severely affecting the transmissions of $U_{A}$. Existing CR schemes can be broadly classified as either interweave, underlay, or overlay [3, 5-17]. For convenience in the following discussions, let $T_{A}$ and $R_{A}$ denote the primary transmitter and primary receiver, respectively, and let $T_{B}$ and $R_{B}$

\footnotetext{
*Correspondence: weilunlin@mail.fcu.edu.tw.

1 Department of Communications Engineering, Feng Chia University,

Taichung, 40724 Taiwan

Full list of author information is available at the end of the article
}

denote the secondary transmitter and secondary receiver, respectively.

In interweave networks, $U_{B}$ transmits its signal to the intended receiver only if the spectrum is not occupied by $U_{A}$. The performance of the interweave $\mathrm{CR}$ schemes is thus dominated by the ability of the users to sense the spectrum occupancy $[11,12]$. If the users fail to detect spectrum holes, performance degradation inevitably occurs. Thus, spectrum detection is a critical issue in interweave CR networks. Furthermore, each user consumes a distinct channel in the interweave CR model, and hence the capacity of the system to accommodate users is restricted. The underlay CR model improves spectrum utilization by allowing $U_{B}$ to convey its signal over the same channel as that used by $U_{A}$ provided that the transmissions of $T_{B}$ do not interfere with those of $R_{A}$ too severely. To minimize interference at $R_{A}, T_{B}$ should be designed such that the power of its interference falls below a certain predefined threshold [2, 3, 13-15]. However, the error performance of $R_{B}$ may still be unsatisfactory due to additional constraints imposed at $R_{A}$. It is noted that this effect is particularly apparent when $T_{B}$ is located physically close to $R_{A}$.

\section{是 Springer}

(C) 2015 Lin et al. Open Access This article is distributed under the terms of the Creative Commons Attribution 4.0 International License (http://creativecommons.org/licenses/by/4.0/), which permits unrestricted use, distribution, and reproduction in any medium, provided you give appropriate credit to the original author(s) and the source, provide a link to the Creative Commons license, and indicate if changes were made. 
The overlay CR model is designed to overcome the poor error performance at $R_{B}$ by enabling $T_{B}$ to help $U_{A}$ relay its signal while concurrently transmitting its own signal. This assistive function can be realized using either some form of cooperative relaying technique or a superposition coding (SC) scheme $[5,14-17]$. For the case where $T_{B}$ is equipped with multiple antennas [6], the zero-forcing beamformer can be used to convey the signals of $U_{A}$ and $U_{B}$ concurrently using a random vector quantization feedback mechanism $[5,14]$. In [7], the primary and secondary systems operate simultaneously using the space-time code originally developed for multiple-input multiple-output systems. More specifically, Alamouti code is applied at the secondary transmitter such that the signals received at the receiver can be decoupled and interference-free signals obtained. The overlay designs in $[5-7,14,18]$, rely on either precoding or the use of space-time coding at $T_{B}$. However, for some applications such as sensor and body networks, allocating single antenna at each node can practically save power and cost $[19,20]$.

It was shown in [21] and [19] that through the assistance of $T_{B}$ the error performance of $U_{A}$ can be enhanced such that the required quality-of-service (QoS) is guaranteed. However, an efficient CR design is crucial when space diversity is not available at the transceiver pairs of $U_{A}$ and $U_{B}$. Accordingly, this study focuses specifically on the problem of QoS-controlled overlay CR design with no space diversity. In developing the proposed overlay design, an assumption is made that the signals are transmitted in two phases, as shown in Fig. 1 . In the first phase, $T_{A}$ broadcasts its signal to both $R_{A}$ and $T_{B}$. In the second phase, $T_{B}$ performs decode-and-forward (DF) over the received signal from $T_{A}$. Specifically, if $T_{B}$ fails to decode the received signal from $T_{A}, T_{B}$ transmits only its own signal. However, if decoding is successful, $T_{B}$ transmits an SC-combined signal comprising the signals of both $U_{A}$ 's and $U_{B}$ 's signals to $R_{A}$ and $R_{B}$ with an appropriate power gain. $R_{A}$ combines the two signals received from $T_{A}$ and $T_{B}$ using a maximum ratio combining (MRC) technique and then decodes the signal. Similarly, $R_{B}$ decodes the SC-combined signal from $T_{B}$.

Due to the signal transmission protocol used in the considered overlay CR network, the error performances of $U_{A}$ and $U_{B}$ are inherently affected by both the transmitted powers of $T_{A}$ and $T_{B}$ and the power allocation factor used in the $\mathrm{SC}$ scheme. To evaluate the respective effects of these factors on the error performance, this study commences by analyzing the BERs of $U_{A}$ and $U_{B}$, where the BER is expressed as a function of the transmitted powers of $U_{A}$ and $U_{B}$ and the power allocation ratio applied in the $\mathrm{SC}$ scheme. A power allocation strategy for the SC scheme is then proposed to minimize the total power consumption of the two users while simultaneously satisfying their respective BER constraints (i.e., QoS requirements). The analytical BER formulations are not convex, and hence determining the optimal solution for the power allocation ratio is challenging, even for numerical methods. To address this problem, two more tractable BER formulas are proposed for $U_{A}$ 's and $U_{B}$ 's BER. The optimum power allocation factor is then determined by solving these two approximations with the proposed non-iterative and iterative approaches. The validity of the proposed power allocation strategy under various channel link conditions is demonstrated by means of numerical simulations.

The remainder of this paper is organized as follows. Section 2 introduces the signal model of the overlay transceiver structure and derives the related analytical BER formulations. The approximated BER expressions are then formulated. Section 3 describes the proposed optimal power allocation strategy based on the approximated BER expressions. Section 4 presents and discusses the simulation results. Finally, Section 5 provides some brief concluding remarks.

\section{System model and BER performance}

\subsection{System model}

Figure 1 shows the overlay spectrum-sharing system considered in the present study. Note that each node is

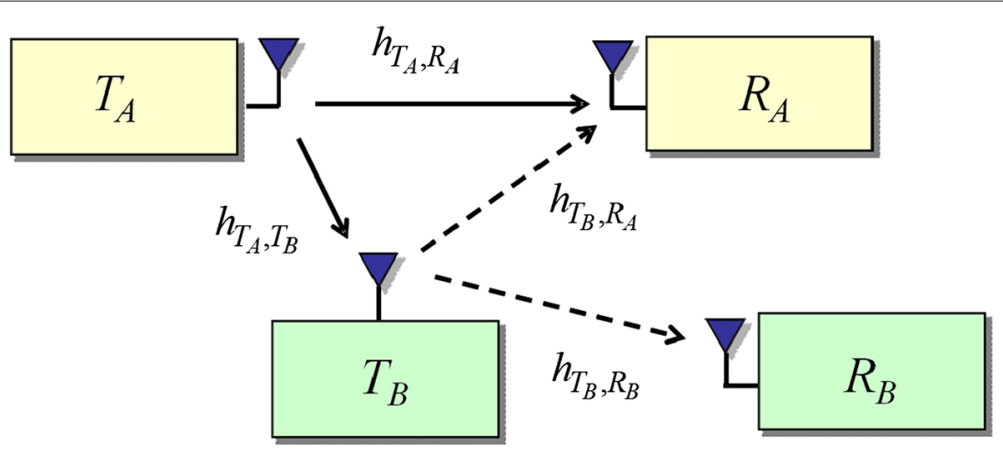

Fig. 1 Cooperative spectrum sharing system with single antenna equipped at each node 
equipped with only a single antenna. The received signals $y_{R_{A}}^{(1)} \in \mathbb{C}$ and $y_{T_{B}}^{(1)} \in \mathbb{C}$ at $R_{A}$ and $T_{B}$ in the first phase can be expressed respectively as

$$
y_{R_{A}}^{(1)}=h_{T_{A}, R_{A}} \sqrt{P_{A}} s_{A}+n_{R_{A}}^{(1)}
$$

and

$$
y_{T_{B}}^{(1)}=h_{T_{A}, T_{B}} \sqrt{P_{A}} s_{A}+n_{T_{B}}^{(1)} .
$$

In (1) and (2), $s_{A}$ denotes the signal transmitted by $U_{A}$. Moreover, $h_{T_{A}, R_{A}} \sim \mathcal{G}_{0}\left(\sigma_{T_{A}, R_{A}}^{2}\right)$ and $h_{T_{A}, T_{B}} \sim$ $\mathcal{G}_{0}\left(\sigma_{T_{A}, T_{B}}^{2}\right)$ are the channel gains of links $T_{A}$-to- $R_{A}$ and $T_{A}$-to- $T_{B}$, respectively, with variance $\sigma_{T_{A}, R_{A}}^{2}$ and $\sigma_{T_{A}, T_{B}}^{2}$. Here, $\mathcal{G}_{0}\left(\sigma^{2}\right)$ denotes a zero-mean complex Gaussian distribution with variance $\sigma^{2}$; finally, $n_{R_{A}}^{(1)} \sim \mathcal{G}_{0}\left(\sigma_{n, R_{A}}^{2}\right)$ and $n_{T_{B}}^{(1)} \sim \mathcal{G}_{0}\left(\sigma_{n, T_{B}}^{2}\right)$ denote the noise received at $R_{A}$ and $T_{B}$, respectively, with variance $\sigma_{n, R_{A}}^{2}$ and $\sigma_{n, T_{B}}^{2}$; and $P_{A}$ is the transmitted power of $T_{A}$.

When $T_{B}$ receives the signal from $T_{A}$ in the first phase, it performs a DF operation on the received signal $s_{A}$. If $s_{A}$ is decoded correctly, then $T_{B}$ combines $s_{A}$ and $s_{B}$ using $\mathrm{SC}^{1}$. However, if the decoding process fails, then $T_{B}$ simply transmits its own signal $s_{B}$. Note that in the present study, it is assumed $R_{A}$ learns the result of the DF operation through a control signal. Thus, the received signal at $R_{A}$ and $R_{B}$ in the second phase are expressed respectively as

$y_{R_{A}}^{(2)}= \begin{cases}h_{T_{B}, R_{A}} \sqrt{P_{B}}\left(\sqrt{\delta} s_{A}+\sqrt{1-\delta} s_{B}\right)+n_{R_{A}}^{(2)} & , \text { if } \mathrm{T}_{\mathrm{B}} \text { decodes signal correctly } \\ 0 & \text {, otherwise }\end{cases}$

$y_{R_{B}}^{(2)}= \begin{cases}h_{T_{B}, R_{B}} \sqrt{P_{B}}\left(\sqrt{\delta} s_{A}+\sqrt{1-\delta} s_{B}\right)+n_{R_{B}}^{(2)}, & \text { if } \mathrm{T}_{\mathrm{B}} \text { decodes signal correctly } \\ h_{T_{B}, R_{B}} \sqrt{P_{B}} s_{B}+n_{R_{B}}^{(2)} & \text {, otherwise. }\end{cases}$

Here, $h_{T_{B}, R_{A}} \sim \mathcal{G}_{0}\left(\sigma_{T_{B}, R_{A}}^{2}\right)$ and $h_{T_{B}, R_{B}} \sim \mathcal{G}_{0}\left(\sigma_{T_{B}, R_{B}}^{2}\right)$ are the fading gains of links $T_{B}$-to- $R_{A}$ and $T_{B}$-to- $R_{B}$ respectively, with variance $\sigma_{T_{B}, R_{A}}^{2}$ and $\sigma_{T_{B}, R_{B}}^{2} ; n_{R_{A}}^{(2)} \sim$ $\mathcal{G}_{0}\left(\sigma_{n, R_{A}}^{2}\right)$ and $n_{R_{B}}^{(2)} \sim \mathcal{G}_{0}\left(\sigma_{n, R_{B}}^{2}\right)$ denote the noise received at $R_{A}$ and $R_{B}$, respectively, with variance $\sigma_{n, R_{A}}^{2}$ and $\sigma_{n, R_{B}}^{2} ; P_{B}$ is the transmitted power of $T_{B}$; and $\delta$ is the power allocation factor applied for combining signals $s_{A}$ and $s_{B}$ in the SC scheme. The larger $\delta$ is the larger portion of the composite signal allocating to $s_{A}$.

\subsection{BER performance}

In evaluating the BER performance of the considered overlay CR scheme, the following discussions commence by deriving analytical formulas for the BERs in decoding $s_{A}$ at $R_{A}$ and $s_{B}$ at $R_{B}$, respectively. Tight approximated
BER expressions are then derived to facilitate the SC power allocation optimization process.

\subsubsection{BER performance in decoding $s_{A}$ for $U_{A}$}

Decoding signal $s_{A}$ at $R_{A}$ involves two diverse cases, namely $T_{B}$ decodes $s_{A}$ correctly and $T_{B}$ decodes $s_{A}$ incorrectly. For the former case, $T_{B}$ transmits the $\mathrm{SC}$-combined signal shown in (3) and (4) to $R_{A}$ and $R_{B}$ concurrently in the second phase. It is thus necessary to first decode $s_{B}$ correctly and then to subtract $s_{B}$ from $y_{R_{A}}^{(2)}$. Since $s_{B}$ is subtracted first, the SC power allocation factor $\delta$ is restricted to the interval $[0,0.5]$ to avoid serious degradation in decoding $s_{A}$. Then, MRC is adopted to combine the extracted signals for decoding $s_{A}$ from the two phases. Note that if $R_{A}$ fails to decode $s_{B}$, MRC is not performed, and $s_{A}$ is decoded merely based on the signal $y_{R_{A}}^{(1)}$. Here, we denote $P_{e, s_{A}}^{R_{A}, I}$ as the BER of $s_{A}$ at $R_{A}$ for this case.

For the latter case, $T_{B}$ incorrectly decodes $s_{A}$, where $R_{A}$ detects $s_{A}$ through $y_{R_{A}}^{(1)}$. Denote $P_{e, s_{A}}^{T_{B}}$ and $P_{e, s_{A}}^{R_{A}, I I}$ as the BERs of $s_{A}$ at $T_{B}$ and $R_{A}$, respectively. The overall average BER denoted as $P_{e, A}$ can then be evaluated as

$$
P_{e, A}=P_{e, s_{A}}^{T_{B}} P_{e, s_{A}}^{R_{A}, I I}+\left(1-P_{e, s_{A}}^{T_{B}}\right) P_{e, s_{A}}^{R_{A}, I} .
$$

The three BER terms in (5), i.e., $P_{e, S_{A}}^{T_{B}}, P_{e, s_{A}}^{R_{A} I}$, and $P_{e, s_{A}}^{R_{A}, I I}$ are separately derived in the following. Without loss of generality, for $s_{A}$ and $s_{B}$, binary phase shift keying is adopted and an equal probability of the data symbol outcomes is assumed.

Since $s_{A}$ is corrupted only by the fading channel gain $h_{T_{A}, R_{A}}$, the conditioned BER can be expressed as [22]

$$
P_{e, S_{A}}^{T_{B}}\left(h_{T_{A}, T_{B}}\right)=Q\left(\sqrt{2\left(\frac{P_{A}\left|h_{T_{A}, T_{B}}\right|^{2}}{\sigma_{n, R_{A}}^{2}}\right)}\right) .
$$

The channel gains are assumed to be complex Gaussian distributed. As a result, $P_{e, s_{A}}^{T_{B}}$ can be easily obtained by averaging $P_{e, s_{A}}^{T_{B}}\left(h_{T_{A}, T_{B}}\right)$ over $h_{T_{A}, T_{B}}$ to give [22]

$$
\begin{aligned}
P_{e, S_{A}}^{T_{B}} & =E\left\{P_{e, S_{A}}^{T_{B}}\left(h_{T_{A}, T_{B}}\right)\right\} \\
& =\frac{1}{2}\left[1-\sqrt{\frac{\gamma_{T_{A}, T_{B}}}{1+\gamma_{T_{A}, T_{B}}}}\right]
\end{aligned}
$$

with $\gamma_{T_{A}, T_{B}}=\sigma_{T_{A}, T_{B}}^{2} P_{A} / \sigma_{n, T_{B}}^{2}$ and $E\{\cdot\}$ being expectation operation.

Similarly, $P_{e, s_{A}}^{R_{A}, I I}$ can be obtained as [22]

$$
P_{e, s_{A}}^{R_{A}, I I}=\frac{1}{2}\left[1-\sqrt{\frac{\gamma_{T_{A}, R_{A}}}{1+\gamma_{T_{A}, R_{A}}}}\right]
$$

where $\gamma_{T_{A}, R_{A}}=\sigma_{T_{A}, R_{A}}^{2} P_{A} / \sigma_{n, R_{A}}^{2}$.

Calculating $P_{e, s_{A}, I}^{R_{A}}$ also involves two diverse cases, namely, $s_{B}$ is correctly decoded at $R_{A}$ in the second phase, or $s_{B}$ is incorrectly decoded. For the former case, if the 
received signal $y_{R_{A}}^{(2)}$ subtracts the correctly decoded $s_{B}$ to be $\tilde{y}_{R_{A}}^{(2)}$, then an MRC operation is optimally performed over the received signals $y_{R_{A}}^{(1)}$ and $\tilde{y}_{R_{A}}^{(2)}{ }^{2}$ Since $s_{B}$ needs to be correctly decoded first, $s_{B}$ requires a larger amount of power to be allocated, which means $0 \leq \delta \leq 0.5$. Otherwise, the error occurs even when the noise is absent. Let the BER of this case be denoted as $P_{e, s_{A}}^{R_{A}, I \text { (MRC) }}$. The corresponding BER conditioned on the channel gains required for decoding $s_{A}$ can be derived as

$P_{e, s_{A}}^{R_{A}, I,(\mathrm{MRC})}\left(h_{T_{A}, R_{A}}, h_{T_{B}, R_{A}}\right)=Q\left(\sqrt{2\left(\frac{P_{A}\left|h_{T_{A}, R_{A}}\right|^{2}}{\sigma_{n, R_{A}}^{2}}+\frac{P_{B}\left|h_{T_{B}, R_{A}}\right|^{2} \delta}{\sigma_{n, R_{A}}^{2}}\right)}\right)$.

For the latter case, if $s_{B}$ is decoded incorrectly, we only use $y_{R_{A}}^{(1)}$ to detect $s_{A}$, and the corresponding BER is equal to $P_{e, s_{A}}^{R_{A}, I I}$ as shown in (8). Let the BER of $s_{B}$ at $R_{A}$ be denoted as $P_{e, s_{B}}^{R_{A}, I}$ with the given channel gain $h_{T_{B}, R_{A}}, P_{e, s_{B}}^{R_{A}, I}$ can then be computed as

$$
\begin{aligned}
P_{e, s_{B}}^{R_{A}, I}\left(h_{T_{B}, R_{A}}\right)= & \sum_{i, j=0}^{1} P_{e, s_{B}}^{R_{A}, I}\left(h_{T_{B}, R_{A}}, s_{A}=(-1)^{i}, s_{B}=(-1)^{j}\right) \\
& \times P\left(s_{A}=(-1)^{i}\right) P\left(s_{B}=(-1)^{j}\right) \\
= & \frac{1}{2} \sum_{i=0}^{1} P_{e, s_{B}}^{R_{A}, I}\left(h_{T_{B}, R_{A}}, s_{A}=(-1)^{i}, s_{B}=1\right) \\
= & \frac{1}{2} \sum_{i=0}^{1} Q\left(\sqrt{2 \frac{P_{B}\left|h_{T_{B}, R_{A}}\right|^{2}}{\sigma_{n, R_{A}}^{2}}\left(\sqrt{1-\delta}+(-1)^{i} \sqrt{\delta}\right)^{2}}\right) .
\end{aligned}
$$

Here, we can observe that the formulation of (10) is more complicated than that of (6) since $s_{B}$ is decoded from a composite signal in (3), while $s_{A}$ is decoded from the received signal in (2). The BER in decoding $s_{A}$ at $R_{A}$ can be obtained with the assistance of (8)-(10) as

$$
\begin{aligned}
& P_{e, s_{A}}^{R_{A}, I}=\left(1-P_{e, s_{B}}^{R_{A}, I}\right) P_{e, s_{A}}^{R_{A}, I,(M R C)}+P_{e, s_{B}}^{R_{A}, I} P_{e, s_{A}}^{R_{A}, I I} \\
& =\int_{0}^{\infty} \int_{0}^{\infty}\left\{\left(1-P_{e, s_{B}}^{R_{A}, I}\left(h_{T_{B}, R_{A}}\right)\right) P_{e, s_{A}}^{R_{A}, I,(M R C)}\left(h_{T_{A}, R_{A}}, h_{T_{B}, R_{A}}\right)\right. \\
& \left.+P_{e, s_{B}}^{R_{A}, I}\left(h_{T_{B}, R_{A}}\right) P_{e, s_{A}}^{R_{A}, I}\left(h_{T_{A}, R_{A}}\right)\right\} \\
& e^{-\frac{\left|h_{T_{A}, R_{A}}\right|^{2}}{\gamma_{T_{A}, R_{A}}}-\frac{\left|h_{T_{B}, R_{A}}\right|^{2}}{\gamma_{T_{B}, R_{A}}}} d\left|h_{T_{A}, R_{A}}\right|^{2} d\left|h_{T_{B}, R_{A}}\right|^{2}
\end{aligned}
$$

where $\gamma_{T_{A}, R_{A}}=\sigma_{T_{A}, R_{A}}^{2} P_{A} / \sigma_{n, R_{A}}^{2}$ and $\gamma_{T_{B}, R_{A}}=$ $\sigma_{T_{B}, R_{A}}^{2} P_{B} / \sigma_{n, R_{A}}^{2}$. Finally, (5) can be calculated in the close form using the approximation given in Appendix A, where $P_{e, A}$ is obtained as a function of $\delta, P_{A}$, and $P_{B}$.

\subsubsection{BER performance in decoding $s_{B}$ for $U_{B}$}

Determining the BER of $U_{B}$ also requires the consideration of two separate cases, namely $T_{B}$ decodes $s_{A}$ correctly and incorrectly. First, consider the case where $T_{B}$ decodes $s_{A}$ correctly. In this scenario, $R_{B}$ receives an $\mathrm{SC}$-combined composite signal from $T_{B}$ in the second phase. Consequently, $s_{B}$ can be directly decoded from the composite signal $y_{R_{B}}^{(2)}$ using (4). As for the derivation of $P_{e, s_{B}}^{R_{A}, I}\left(h_{T_{B}, R_{A}}\right)$ in (10), the conditional BER in decoding $s_{B}$ at $R_{B}$, denoted as $P_{e, s_{B}}^{R_{B}, I}\left(h_{T_{B}, R_{B}}\right)$, is given by

$$
P_{e, S_{B}}^{R_{B}, I}\left(h_{T_{B}, R_{B}}\right)=\frac{1}{2} \sum_{i=0}^{1} Q\left(\sqrt{2 \frac{P_{B}\left|h_{T_{B}, R_{B}}\right|^{2}}{\sigma_{n, R_{B}}^{2}}\left(\sqrt{1-\delta}+(-1)^{i} \sqrt{\delta}\right)^{2}}\right) .
$$

Averaging (12) over $\left|h_{T_{B}, R_{B}}\right|^{2}$ with exponential distribution, $P_{e, s_{B}}^{R_{B}, I}$ is obtained as

$$
\begin{aligned}
P_{e, S_{B}}^{R_{B}, I} & =\int_{0}^{\infty} P_{e, s_{B}}^{R_{B}, I}\left(h_{T_{B}, R_{B}}\right) e^{-\frac{\left|h_{T_{B}, R_{B}}\right|^{2}}{\gamma_{T_{B}, R_{B}}}} d\left|h_{T_{B}, R_{B}}\right|^{2} \\
& =\frac{1}{2} \sum_{i=0}^{1} \frac{1}{2}\left[1-\sqrt{\frac{\left(\sqrt{1-\delta}+(-1)^{i} \sqrt{\delta}\right)^{2} \gamma_{T_{B}, R_{B}}}{1+\left(\sqrt{1-\delta}+(-1)^{i} \sqrt{\delta}\right)^{2} \gamma_{T_{B}, R_{B}}}}\right]
\end{aligned}
$$

with $\gamma_{T_{B}, R_{B}}=\sigma_{T_{B}, R_{B}}^{2} P_{B} / \sigma_{n, R_{B}}^{2}$.

For the case where $T_{B}$ decodes $s_{A}$ incorrectly, $T_{B}$ merely transmits $s_{B}$ to $R_{B}$. Thus, the BER in decoding $s_{B}$, denoted as $P_{e, s_{B}}^{R_{B}, I I}$, can then be calculated as

$$
P_{e, s_{B}}^{R_{B}, I I}=\frac{1}{2}\left[1-\sqrt{\frac{\gamma_{T_{B}, R_{B}}}{1+\gamma_{T_{B}, R_{B}}}}\right] .
$$

Finally, the BER in decoding $s_{B}$ at $R_{B}$, denoted by $P_{e, B}$, can be determined from the BERs given above as

$$
\begin{aligned}
P_{e, B}= & \left(1-P_{e, s_{A}}^{T_{B}}\right) P_{e, s_{B}}^{R_{B}, I}+P_{e, s_{A}}^{T_{B}} P_{e, s_{B}}^{R_{B}, I I} \\
= & \frac{1}{8}\left(1+\sqrt{\frac{\gamma_{T_{A}, T_{B}}}{1+\gamma_{T_{A}, T_{B}}}}\right) \\
& \times\left(\sum_{i=0}^{1}\left(1-\sqrt{\frac{\left(\sqrt{1-\delta}+(-1)^{i} \sqrt{\delta}\right)^{2} \gamma_{T_{B}, R_{B}}}{1+\left(\sqrt{1-\delta}+(-1)^{i} \sqrt{\delta}\right)^{2} \gamma_{T_{B}, R_{B}}}}\right)\right) \\
& +\frac{1}{4}\left(1-\sqrt{\frac{\gamma_{T_{A}, T_{B}}}{1+\gamma_{T_{A}, T_{B}}}}\right)\left(1-\sqrt{\frac{\gamma_{T_{B}, R_{B}}}{1+\gamma_{T_{B}, R_{B}}}}\right)
\end{aligned}
$$

which is also a function of $\delta, P_{A}$, and $P_{B}$.

\subsubsection{Approximate BER expressions}

To facilitate the power allocation optimization process, this section derives two more tractable approximations for $P_{e, A}$ and $P_{e, B}$, denoted as $\widetilde{P}_{e, A}$ and $\widetilde{P}_{e, B}$, respectively. 
The BER $P_{e, A}$ derived in (5) can be approximated as

$$
\begin{aligned}
P_{e, A} \approx & \widetilde{P}_{e, A}=\left(\frac{\sigma_{n, T_{B}}^{2}}{4 \sigma_{T_{A}, T_{B}}^{2} P_{A}}\right) \times\left(\frac{\sigma_{n, R_{A}}^{2}}{4 \sigma_{T_{A}, R_{A}}^{2} P_{A}}\right) \\
& +\left(1-\frac{\sigma_{n, T_{B}}^{2}}{4 \sigma_{T_{A}, T_{B}}^{2} P_{A}}\right) \\
& \times\left(\frac{\sigma_{n, R_{A}}^{4}}{16 \sigma_{T_{A}, R_{A}}^{2} \sigma_{T_{B}, R_{A}}^{2} P_{A} P_{B}}\left[\frac{1}{(1-2 \delta)^{2}}+\frac{3}{\delta}\right]\right) .
\end{aligned}
$$

Note that the detailed derivation is provided in Appendix A.

Meanwhile, BER $P_{e, B}$ derived in (15) can be approximated as

$$
\begin{aligned}
P_{e, B} & \approx \widetilde{P}_{e, B} \\
& =\left(\frac{\sigma_{n, T_{B}}^{2}}{4 \sigma_{T_{A}, T_{B}}^{2} P_{A}}\right) \times\left(\frac{\sigma_{n, R_{B}}^{2}}{4 \sigma_{T_{B}, R_{B}}^{2} P_{B}}\right)+\left(1-\frac{\sigma_{n, T_{B}}^{2}}{4 \sigma_{T_{A}, T_{B}}^{2} P_{A}}\right) \\
& \times\left(\frac{\sigma_{n, R_{B}}^{2}}{4 \sigma_{T_{B}, R_{B}}^{2} P_{B}} \frac{1}{(1-2 \delta)^{2}}\right) .
\end{aligned}
$$

The detailed derivation is provided in Appendix B.
Figure 2 compares the approximated BER curves with true BER curves for $\sigma_{T_{A}, T_{B}}^{2} / \sigma_{n, T_{B}}^{2}=5 \mathrm{~dB}, \sigma_{T_{A}, R_{A}}^{2} / \sigma_{n, R_{A}}^{2}=0$ $\mathrm{dB}, \sigma_{T_{B}, R_{A}}^{2} / \sigma_{n, R_{A}}^{2}=10 \mathrm{~dB}, \sigma_{T_{B}, R_{B}}^{2} / \sigma_{n, R_{B}}^{2}=10 \mathrm{~dB}, P_{A}=P_{B}=1$, and $\delta=0.1$ and 0.3. As shown, the approximated BER values are in good agreement with the actual values; particularly in the high SNR region. Notably, the BER of $U_{A}$ has a diversity gain of two, whereas that of $U_{B}$ has a diversity gain of one. The lower diversity gain of $U_{B}$ arises since it only uses a link to transmit signals.

\section{Optimal power allocation}

\subsection{Optimization of power allocation}

The previous section has analyzed the BER performance of the primary and secondary users in the considered overlay $C R$ network. Observing the analytical results in (5) and (15), it is seen that the BERs for $U_{A}$ and $U_{B}$ are closely related to the transmitted power, i.e., $P_{A}$ and $P_{B}$ and the SC power allocation factor $\delta$. Taking the BER as the benchmark of the provided QoS, this section proposes a strategy for determining the SC allocation factor which minimizes the total power consumption of the two users, $P_{A}+P_{B}$, while simultaneously achieving $P_{e, A} \leq \mathrm{BER}_{A}$ and $P_{e, B} \leq \mathrm{BER}_{B}$, where $\mathrm{BER}_{A}$ and $\mathrm{BER}_{B}$ are the predefined

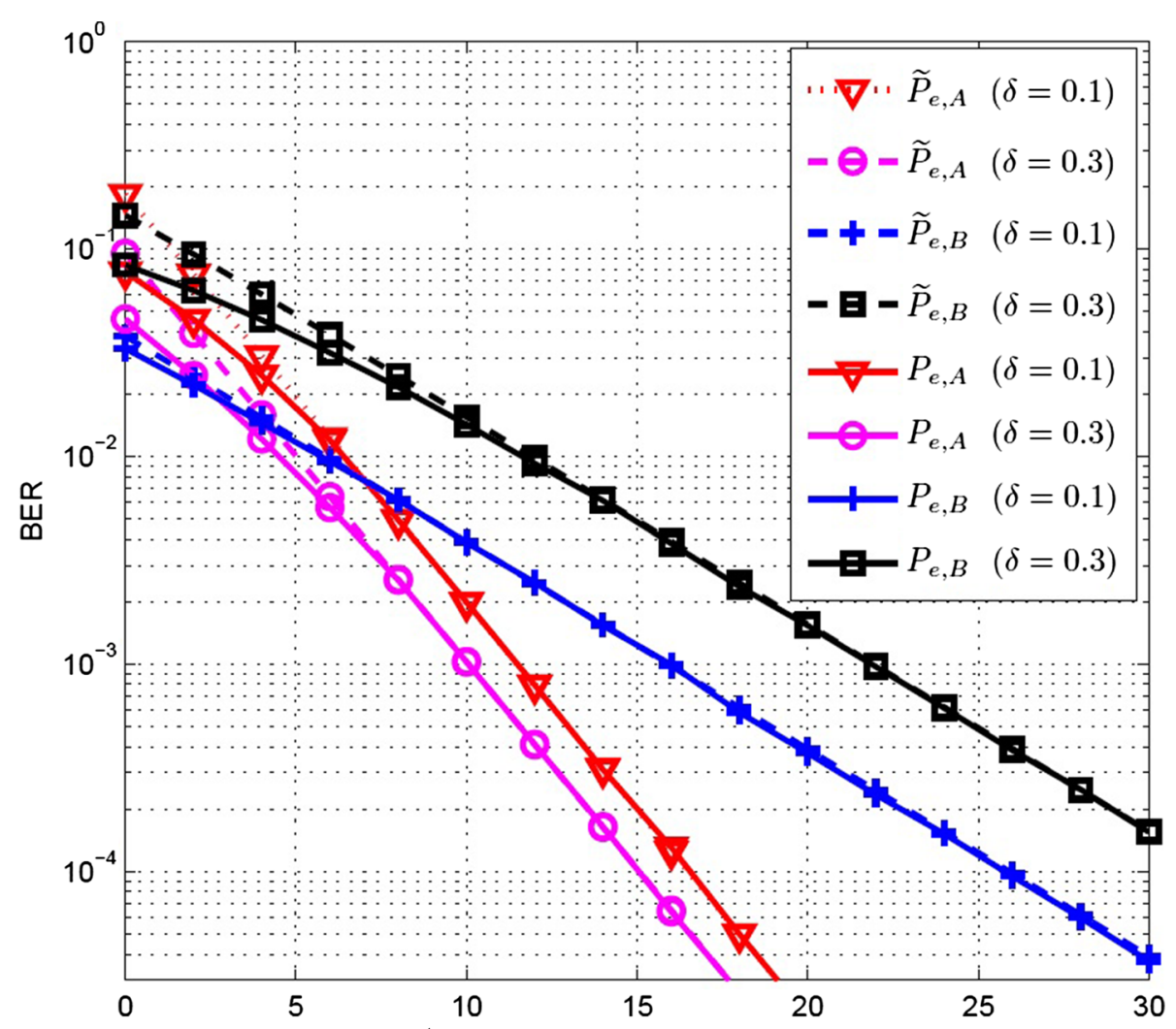

Fig. 2 Comparison of approximated and true BER curves $\left(\sigma_{T_{A}, T_{B}}^{2} / \sigma_{n, T_{B}}^{2}=5, \sigma_{T_{A}, R_{A}}^{2} / \sigma_{n, R_{A}}^{2}=0, \sigma_{T_{B}, R_{A}}^{2} / \sigma_{n, R_{A}}^{2}=\sigma_{T_{B}, R_{B}}^{2} / \sigma_{n, R_{B}}^{2}=10 \mathrm{~dB}, P_{A}=P_{B}=1\right)$ 
QoSs of the two users. The optimization problem can thus be formulated as

$$
\begin{aligned}
& (P 1) \min _{P_{A}, P_{B}, \delta} P_{A}+P_{B} \\
& \text { s.t. } \\
& P_{e, A} \leq \mathrm{BER}_{A}, \quad P_{e, B} \leq \mathrm{BER}_{B}, \\
& P_{A}, P_{B} \geq 0,0 \leq \delta \leq 0.5 .
\end{aligned}
$$

As shown in (5) and (15), the optimization constraints have the form of non-convex functions. Thus, the optimal solution of (18) is almost impossible to obtain, even when using numerical methods. Thus, to facilitate the optimization process, $P_{e_{2} A}$ and $P_{e, B}$ are replaced respectively with $\widetilde{P}_{e, A}$ in (16) and $\widetilde{P}_{e, B}$ in (17). The optimization problem can then be reformulated as

$$
\begin{aligned}
& \text { (P2) } \min _{P_{A}, P_{B}, \delta} P_{A}+P_{B} \\
& \text { s.t. } \\
& \widetilde{P}_{e, A} \leq \mathrm{BER}_{A} \\
& \widetilde{P}_{e, B} \leq \mathrm{BER}_{B} \\
& P_{A}, P_{B} \geq 0,0 \leq \delta \leq 0.5 .
\end{aligned}
$$

The optimal solutions of the proposed optimization $(P 2)$ can be sophisticatedly solved though it is still not convex. We have to mention that the proposed approximations (16) and (17) are conservative approximations meaning that the approximated BERs are the upper bounds of the true BERs, and consequently the QoS requirements are always satisfied. Moreover, the BER approximations are tight when the channel quality of the link $T_{A}$-to$R_{A}$ or $T_{B}$-to- $R_{A}$ is favorable, or equivalently the QoS requirements are assumed to be moderately low. For brevity, define $\eta_{a}=\sigma_{n, R_{A}}^{4} \sigma_{n, T_{B}}^{2} /\left(16 \sigma_{T_{A}, R_{A}}^{2} \sigma_{T_{A}, T_{B}}^{2}\right), \eta_{b}=$ $\sigma_{n, R_{A}}^{4} /\left(16 \sigma_{T_{A}, R_{A}}^{2} \sigma_{T_{B}, R_{A}}^{2}\right), \eta_{c}=\sigma_{n, R_{A}}^{4} \sigma_{n, T_{B}}^{2} /\left(64 \sigma_{T_{A}, R_{A}}^{2}\right.$ $\left.\sigma_{T_{A}, T_{B}}^{2} \sigma_{T_{B}, R_{A}}^{2}\right), \eta_{d}=\sigma_{n, T_{B}}^{2} \sigma_{n, R_{B}}^{2} /\left(16 \sigma_{T_{A}, T_{B}}^{2} \sigma_{T_{B}, R_{B}}^{2}\right), \eta_{e}=$ $\sigma_{n, R_{B}}^{2} /\left(4 \sigma_{T_{B}, R_{B}}^{2}\right)$.

$$
P_{A}=-\frac{g_{A}(\delta)}{3}
$$

To optimize (19), we first notice that $\widetilde{P}_{e, A}$ and $\widetilde{P}_{e, B}$ are monotonically decreasing with increasing $P_{A}$ and/or $P_{B}$ for any fixed $\delta$. Hence, the minimum total transmitted power $\left(P_{A}+P_{B}\right)$ can be achieved solely with $\widetilde{P}_{e, A}$ and $\widetilde{P}_{e, B}$ being satisfied by the minimum requirement of BER, i.e., $\widetilde{P}_{e, A}=\mathrm{BER}_{A}$ and $\widetilde{P}_{e, B}=\mathrm{BER}_{B}$. With these two equalities, (19) can be solved by optimizing the parameter $\delta$ only. Specifically, with $\widetilde{P}_{e, B}=\mathrm{BER}_{B}$, we have

$$
\frac{\eta_{d}}{P_{A} P_{B}}+\frac{\eta_{e}}{P_{B}(1-2 \delta)^{2}}-\frac{\eta_{d}}{P_{A} P_{B}(1-2 \delta)^{2}}=\mathrm{BER}_{B} .
$$

Here, $P_{B}$ can be alternatively represented by

$$
P_{B}=\frac{\eta_{d}}{P_{A} \mathrm{BER}_{B}}+\frac{\eta_{e}}{\mathrm{BER}_{B}(1-2 \delta)^{2}}-\frac{\eta_{d}}{P_{A} \mathrm{BER}_{B}(1-2 \delta)^{2}} .
$$

Substituting (21) into the equality, $\widetilde{P}_{e, A}=\mathrm{BER}_{A}$, with some straightforward manipulations, it is shown that

$$
\begin{aligned}
P_{A}^{3} & +\left(\frac{\eta_{d}}{\eta_{e}}\left((1-2 \delta)^{2}-1\right)-\frac{\mathrm{BER}_{B} \eta_{b}}{\mathrm{BER}_{A} \eta_{e}}\left(1+\frac{3(1-2 \delta)^{2}}{\delta}\right)\right) P_{A}^{2} \\
& +\left(\frac{\mathrm{BER}_{B} \eta_{c}}{\mathrm{BER}_{A} \eta_{e}}\left(1+\frac{3(1-2 \delta)^{2}}{\delta}\right)-\frac{\eta_{a}}{\mathrm{BER}_{A}}\right) P_{A} \\
& +\frac{\eta_{a} \eta_{d}}{\mathrm{BER}_{A} \eta_{e}}\left(1-(1-2 \delta)^{2}\right)=0 .
\end{aligned}
$$

The closed-form solution of $P_{A}$ corresponding to (22) has the form [23]

$$
\begin{aligned}
& +\sqrt[3]{\left(\frac{g_{A}(\delta) g_{B}(\delta)}{6}-\frac{g_{A}^{3}(\delta)}{27}-\frac{g_{C}(\delta)}{2}\right)-\sqrt{\left(\frac{g_{A}(\delta) g_{B}(\delta)}{6}-\frac{g_{A}^{3}(\delta)}{27}-\frac{g_{C}(\delta)}{2}\right)^{2}+\left(\frac{g_{B}(\delta)}{3}-\frac{g_{A}^{2}(\delta)}{9}\right)^{3}}} \\
& +\sqrt[3]{\left(\frac{g_{A}(\delta) g_{B}(\delta)}{6}-\frac{g_{A}^{3}(\delta)}{27}-\frac{g_{C}(\delta)}{2}\right)+\sqrt{\left(\frac{g_{A}(\delta) g_{B}(\delta)}{6}-\frac{g_{A}^{3}(\delta)}{27}-\frac{g_{C}(\delta)}{2}\right)^{2}+\left(\frac{g_{B}(\delta)}{3}-\frac{g_{A}^{2}(\delta)}{9}\right)^{3}}}
\end{aligned}
$$


where

$$
\begin{gathered}
g_{A}(\delta)=\frac{\eta_{d}}{\eta_{e}}\left((1-2 \delta)^{2}-1\right)-\frac{\mathrm{BER}_{B} \eta_{b}}{\mathrm{BER}_{A} \eta_{e}}\left(1+\frac{3(1-2 \delta)^{2}}{\delta}\right), \\
g_{B}(\delta)=\frac{\mathrm{BER}_{B} \eta_{c}}{\mathrm{BER}_{A} \eta_{e}}\left(1+\frac{3(1-2 \delta)^{2}}{\delta}\right)-\frac{\eta_{a}}{\mathrm{BER}_{A}}
\end{gathered}
$$

and

$$
g_{C}(\delta)=\frac{\eta_{a} \eta_{d}}{\mathrm{BER}_{A} \eta_{e}}\left(1-(1-2 \delta)^{2}\right)
$$

To simplify (23), define

$$
f_{Q}(\delta)=\frac{g_{A}(\delta) g_{B}(\delta)}{6}-\frac{g_{A}^{3}(\delta)}{27}-\frac{g_{C}(\delta)}{2}
$$

and

$$
f_{R}(\delta)=\frac{g_{B}(\delta)}{3}-\frac{g_{A}^{2}(\delta)}{9} .
$$

(23) can then be reformulated as

$$
P_{A}=-\frac{g_{A}}{3}+\sqrt[3]{f_{Q}+\sqrt{f_{Q}^{2}+f_{R}^{3}}}-\sqrt[3]{\sqrt{f_{Q}^{2}+f_{R}^{3}}-f_{Q}}
$$

Here, we checked that $f_{Q}^{2}(\delta)+f_{R}^{3}(\delta)<0$ and $f_{R}(\delta)<0$. Thus, (29) can be rewritten as

$$
\begin{aligned}
P_{A}= & -\frac{g_{A}}{3}+\sqrt[3]{f_{Q}+\left(f_{Q}^{2}+f_{R}^{3}\right)^{1 / 2}}-\sqrt[3]{-f_{Q}+\left(f_{Q}^{2}+f_{R}^{3}\right)^{1 / 2}} \\
= & -\frac{g_{A}}{3}+\left(\sqrt[6]{f_{Q}^{2}-\left(f_{Q}^{2}+f_{R}^{3}\right)}\right)\left(\cos \frac{1}{3} \theta+j \sin \frac{1}{3} \theta\right) \\
& +\left(\sqrt[6]{f_{Q}^{2}-\left(f_{Q}^{2}+f_{R}^{3}\right)}\right)\left(\cos \frac{1}{3} \theta-j \sin \frac{1}{3} \theta\right) \\
= & -\frac{g_{A}}{3}+2 \sqrt{-f_{R}(\delta)} \cos \frac{1}{3} \theta
\end{aligned}
$$

where $\cos \theta=\frac{f_{Q}(\delta)}{\sqrt{-f_{R}(\delta)}}$. It can be shown that $-\frac{g_{A}(\delta)}{3}$ and $2 \sqrt{-f_{R}(\delta)} \cos \frac{1}{3} \theta$ are both convex in $\delta \in[0,0.5]$. Thus, $P_{A}$ is convex in $\delta \in[0,0.5]$. It is noted that (23) has only one solution which satisfies both constraints since the other two solutions either contradict the basic power assumption or the BER constraint, i.e., $P_{A} \geq 0$ or $\widetilde{P}_{e, A} \geq 1$, respectively.
From (20), $P_{B}$ can be reformulated as

$$
\begin{aligned}
& P_{B}=\frac{1}{\mathrm{BER}_{B}}\left(\frac{\eta_{d}}{P_{A}}+\frac{\eta_{e}}{(1-2 \delta)^{2}}-\frac{\eta_{d}}{P_{A}(1-2 \delta)^{2}}\right) \\
& =\frac{1}{\mathrm{BER}_{B}}\left(-\frac{\eta_{d}}{P_{A}}\left(\frac{1}{(1-2 \delta)^{2}}-1\right)+\frac{\eta_{e}}{(1-2 \delta)^{2}}\right) \\
& =\frac{1}{\mathrm{BER}_{B}}\left(\begin{array}{c}
\frac{\eta_{d}}{P_{A}}+\underbrace{\left(\eta_{e}-\frac{\eta_{d}}{P_{A}}\right)} \underbrace{(1-2 \delta)^{2}}_{\frac{\sigma_{n} R_{B}}{4 \sigma_{T_{B}, R_{B}}}\left(1-\frac{\sigma_{n, T_{B}}^{2}}{4 P_{A} \sigma_{T_{A}, T_{B}}^{2}}\right)} \\
(1)
\end{array}\right) .
\end{aligned}
$$

From the last equality, it follows that $\eta_{e}-\frac{\eta_{d}}{P_{A}}=$ $\frac{\sigma_{n, R_{B}}^{2}}{4 \sigma_{T_{B}, R_{B}}^{2}}\left(1-\frac{\sigma_{n, T_{B}}^{2}}{4 P_{A} \sigma_{T_{A}, T_{B}}^{2}}\right)$. Notably, $P_{B}$ is convex in $\delta \in$ $[0,0.5]$ for any given $P_{A} \geq \frac{\sigma_{n, T_{B}}^{2}}{4 \sigma_{T_{A}, T_{B}}^{2}}$.

Finally, by adding an auxiliary variable $t$ along with the results in (30) and (31), (19) can be rewritten as the following optimization problem:

(P3) $\min _{t, \delta} \frac{1}{\mathrm{BER}_{B}}\left(\frac{\eta_{d}}{t}+\frac{\eta_{e}}{(1-2 \delta)^{2}}-\frac{\eta_{d}}{t(1-2 \delta)^{2}}\right)+t$

s.t.

$$
\begin{aligned}
& -\frac{g_{A}(\delta)}{3}+2 \sqrt{-f_{R}(\delta)} \cos \frac{1}{3} \theta \leq t \\
& 0 \leq \delta \leq 0.5 \\
& \frac{\sigma_{n, T_{B}}^{2}}{4 \sigma_{T_{A}, T_{B}}^{2}} \leq t
\end{aligned}
$$

where $g_{A}(\delta)$ and $f_{R}(\delta)$ are functions of $\delta$ expressed in (24) and (28), respectively. The problem formulated in (32) is essentially a convex optimization problem. Thus, the solutions of $P_{A}$ and $\delta$ can be obtained via numerical methods, such as the simple steepest descent method, while $P_{B}$ can be obtained via (31).

\subsection{Iterative approach}

The previous subsection first simplifies the optimization problem (P1) to (P2) with two equalities $\left(\widetilde{P}_{e, A}=\mathrm{BER}_{A}\right.$ and $\left.\widetilde{P}_{e, B}=\mathrm{BER}_{B}\right)$, and finally presents the compact results in (P3). In this subsection, we intuitively provide an alternative approach to solve (P2) suboptimally. It is clear that when any two parameters are fixed as constants in (P2), the problem becomes a convex and only the residual parameter has to be optimized. Thus, we can divide (P2) into the following three problems. 


$$
\begin{aligned}
& (P 4-1) \min _{P_{A}} P_{A} \\
& \text { s.t. } \\
& \widetilde{P}_{e, A} \leq \mathrm{BER}_{A} \\
& \widetilde{P}_{e, B} \leq \mathrm{BER}_{B} \\
& P_{A} \geq 0 \\
& (P 4-2) \quad \min _{P_{B}} P_{B} \\
& \text { s.t. } \\
& \widetilde{P}_{e, A} \leq \mathrm{BER}_{A} \\
& \widetilde{P}_{e, B} \leq \mathrm{BER}_{B} \\
& P_{B} \geq 0 \\
& (P 4-3) \quad \min _{\delta} \delta \\
& \text { s.t. } \\
& \widetilde{P}_{e, A} \leq \mathrm{BER}_{A} \\
& \widetilde{P}_{e, B} \leq \mathrm{BER}_{B} \\
& 0 \leq \delta \leq 0.5 .
\end{aligned}
$$

Since $(\mathrm{P} 4-1)-(\mathrm{P} 4-3)$ are convex problems, we can iteratively solve the solutions through the $\mathrm{cvx}$ tool [24]. It is noteworthy that the iterative approach can essentially obtain a local solution and is sensitive to the initial condition, while the proposed non-iterative approach has only one local solution which suggests it is also a global solution. Thus, it is expected that the performance of the noniterative approach is superior to the iterative approach. More discussion is provided in the following section.

\section{Simulation results}

This section evaluates the performance of the proposed power allocation method under realistic overlay CR network conditions. In performing the simulations, it is assumed that all of the channel links suffer fading with independent and identically distributed (i.i.d.) Rayleighdistributed amplitudes. Let $\sigma_{n, T_{B}}^{2}=\sigma_{n, R_{B}}^{2}=\sigma_{n, R_{A}}^{2}=1$, $\mathrm{BER}_{A}=10^{-3}$, and $\mathrm{BER}_{B}=5 \times 10^{-3}$. Furthermore, let three levels of channel quality be considered, namely $\left(\sigma_{T_{A}, R_{A}}^{2}, \sigma_{T_{A}, T_{B}}^{2}, \sigma_{T_{B}, R_{A}}^{2}, \sigma_{T_{B}, R_{B}}^{2}\right)=(0,5,10,10)$, $(0,5,20,20),(0,10,30,30) \mathrm{dBm}$. For convenience, let the three cases be referred to as case 1 , case 2 , and case 3 , respectively. Figure 3 shows the results for the variation of the power consumption with the power allocation factor. Note that the circles represent the solution obtained

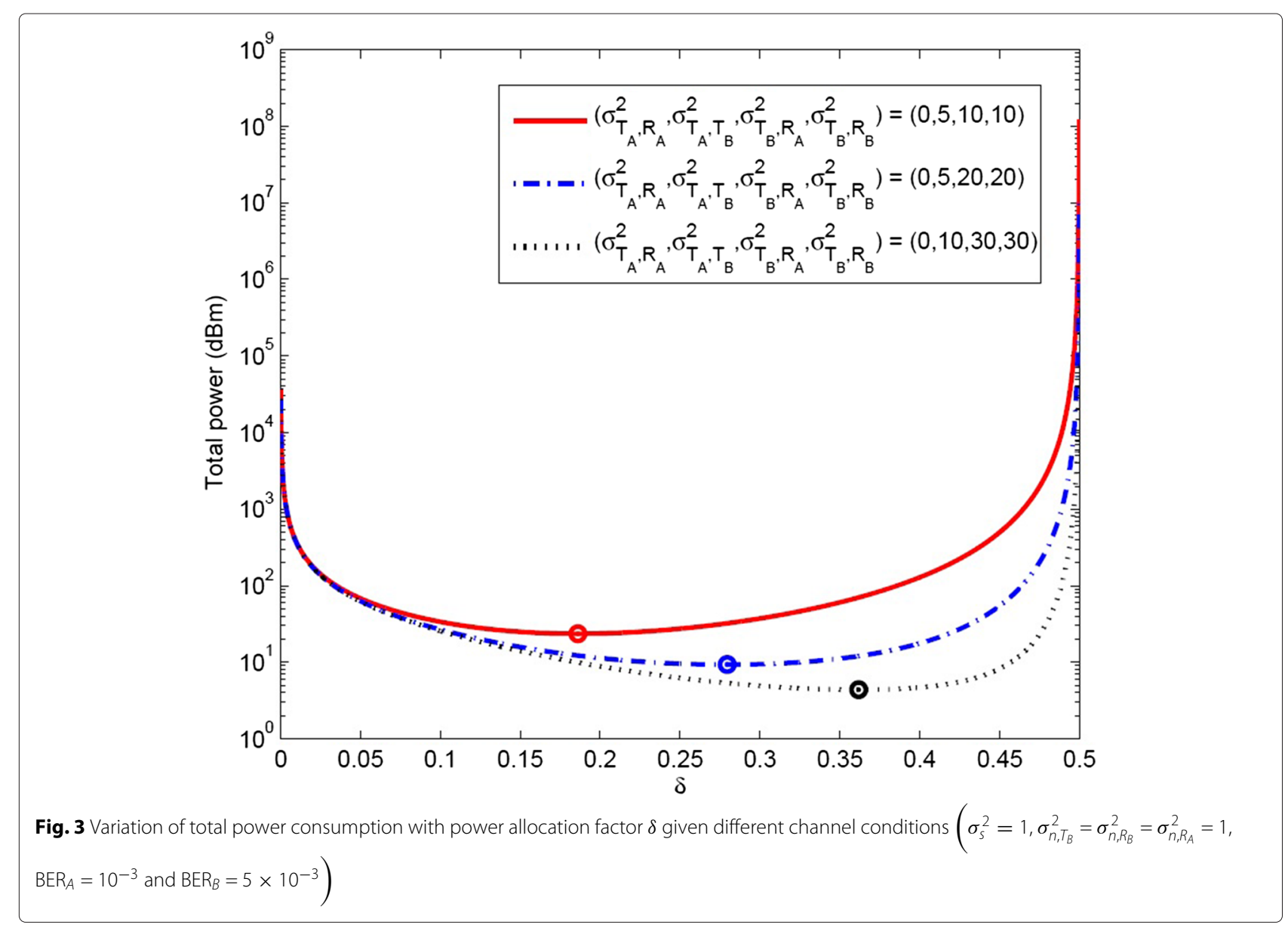


using the proposed method. It is seen that the circles are coincident with the point of minimum total power consumption in every case. In other words, the optimum $\delta$ obtained in (32) coincides perfectly with the numerical results. In addition, it is observed that the total power consumption in case 2 is less than that in case 1 since the channel quality in case 2 is better than that in case 1 . Finally, it is seen that a larger value of $\delta$ results in a higher $\sigma_{T_{B}, R_{B}}^{2}$. This result is intuitively reasonable since when $\sigma_{T_{B}, R_{B}}^{2}$ increases, $\mathrm{BER}_{B}$ is more easily satisfied, and hence a greater amount of power is allocated to $U_{A}$ in the SC scheme.

Further simulations were performed to investigate the variation of the total power consumption associated to the fixed power allocation, the iterative approach, and the proposed design. Let $\sigma_{T_{A}, R_{A}}^{2} / \sigma_{n}^{2}=0 \mathrm{~dB}, \sigma_{T_{A}, T_{B}}^{2} / \sigma_{n}^{2}=15 \mathrm{~dB}$, $\sigma_{T_{B}, R_{B}}^{2} / \sigma_{n}^{2}=\sigma_{T_{B}, R_{A}}^{2} / \sigma_{n}^{2}=\sigma_{h}^{2} / \sigma_{n}^{2}, \delta=0.1,0.2,0.3$, 0.4 . Figures 4 and 5 show the results obtained for the total power consumption and the resultant BERs of $U_{B}$ and $U_{A}$ of the proposed design, respectively. As shown in Fig. 4, for a given $\delta$, the power consumption decreases as $\delta$ decreases. This result follows intuitively since the signal of $U_{A}$ is potentially conveyed through two links, i.e., $T_{A}$-to- $T_{B}$-to- $R_{A}$ and $T_{A}$-to- $R_{A}$, whereas the signal of $U_{B}$ is transmitted through only one link, i.e., $T_{B}$-to- $R_{B}$. Due to the fixed channel quality of $T_{B}$-to- $R_{B}, T_{B}$ is able to use approximated constant power consumption to maintain the BER at $R_{B}$. A smaller value of $\delta$ indicates that $U_{B}$ offers a lower level of assistance. Thus, the power consumption at $T_{A}$ must be increased, and hence the total power consumption also increases. Since the optimum value of $\delta$ is obtained by the proposed SC allocation factor strategy, the minimal power consumption can also be obtained. The iterative approach performs worse than the proposed design since the iterative approach is a suboptimal method. Figure 5 shows the resultant BER corresponding to the proposed design (the curve related to the optimum $\delta$ ) in Fig. 4. The results confirm that the resultant BERs are consistent with the required QoS constants. Since $\delta$ represents the power allocated to $U_{A}$, the power of $U_{A}$ must be increased to enable $R_{A}$ to conduct MRC when $\delta$ is small, which ensures that $R_{A}$ satisfies the QoS constraint.

A final series of simulations was performed to investigate the power consumption given different channel link qualities and QoS constraints. Let $\mathrm{BER}_{A}=$ $\mathrm{BER}_{B}=\gamma$. Four different link qualities were considered, namely $\left(\sigma_{T_{A}, R_{A}}^{2}, \sigma_{T_{A}, T_{B}}^{2}, \sigma_{T_{B}, R_{A}}^{2}, \sigma_{T_{B}, R_{B}}^{2}\right)=(0,5,10,10)$, $(0,5,20,20),(0,10,20,20),(0,15,30,30) \mathrm{dBm}$. As shown in Fig. 6, for a given link quality, the power consumption increases with a decreasing BER. Furthermore, for a constant BER, the power consumption given a channel

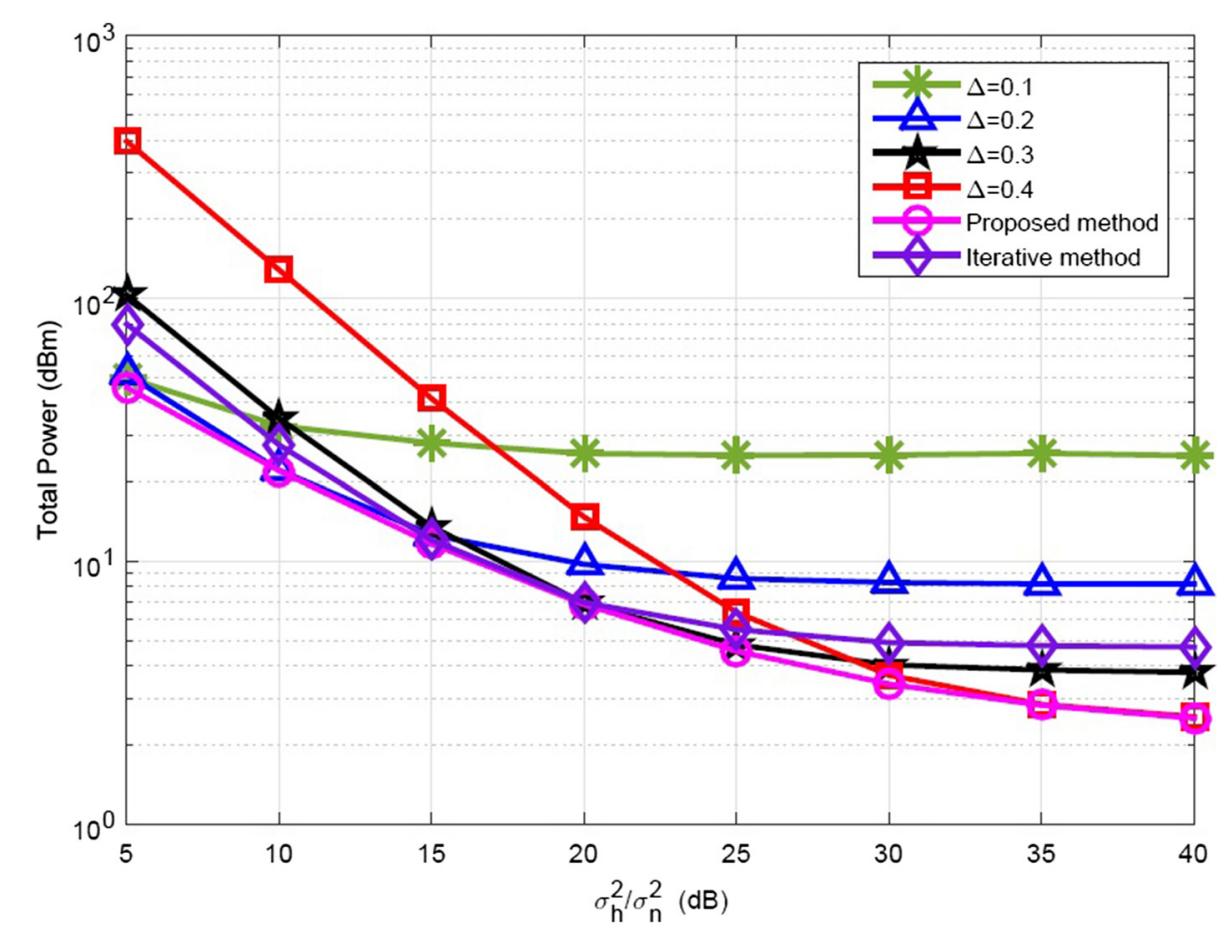

Fig. 4 Total power consumption as function of power allocation factor $(\delta=0.1,0.2,0.3,0.4)$, the iterative approach, and the proposed design. $\left(\sigma_{T_{A}, R_{A}}^{2} / \sigma_{n}^{2}=0 \mathrm{~dB}, \sigma_{T_{A}, T B}^{2} / \sigma_{n}^{2}=15 \mathrm{~dB}, \sigma_{T_{B}, R_{B}}^{2} / \sigma_{n}^{2}=\sigma_{T_{B}, R_{A}}^{2} / \sigma_{n}^{2}=\sigma_{h}^{2} / \sigma_{n}^{2}\right)$ 


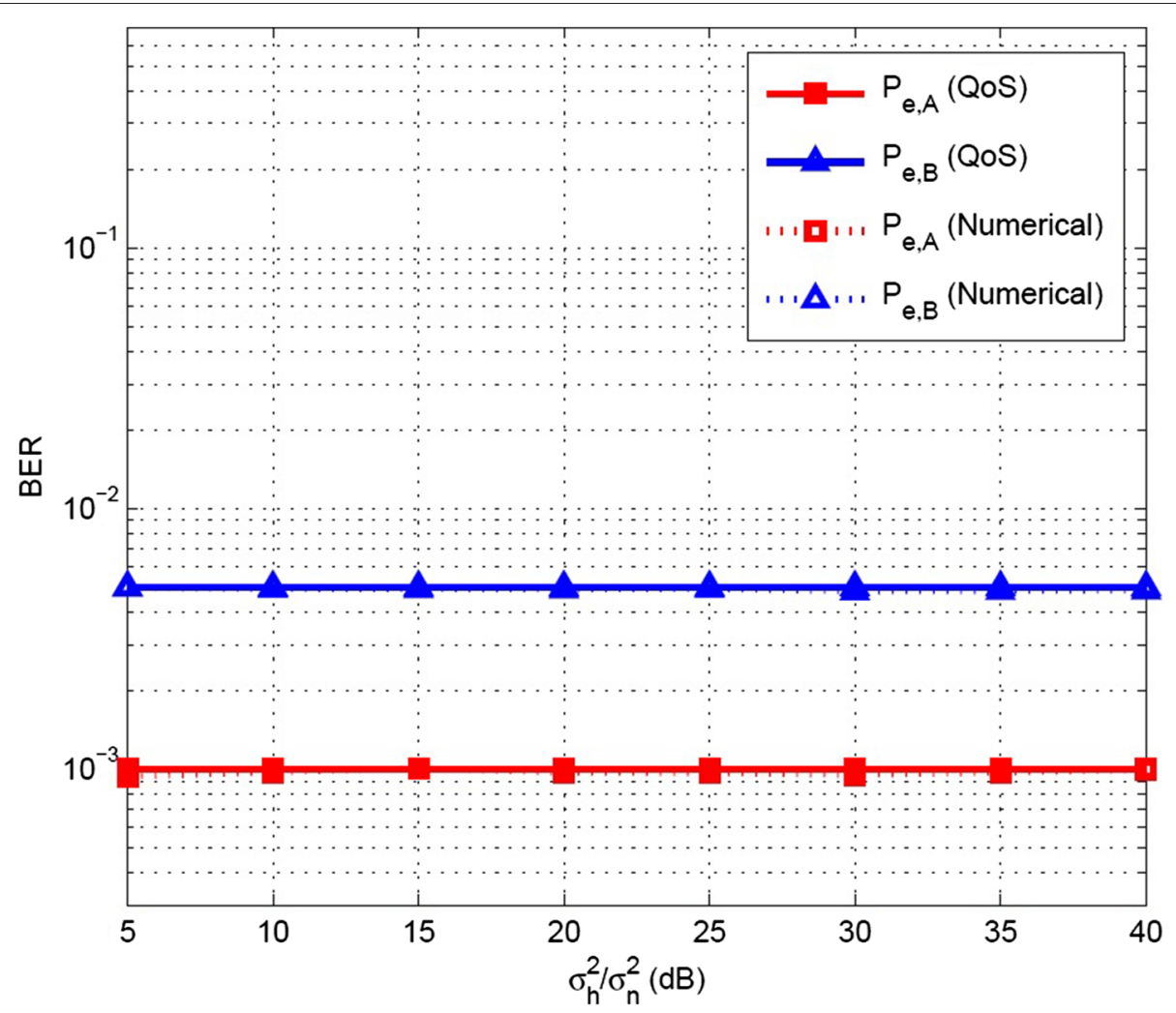

Fig. 5 Resultant BERs corresponding to Fig. 4

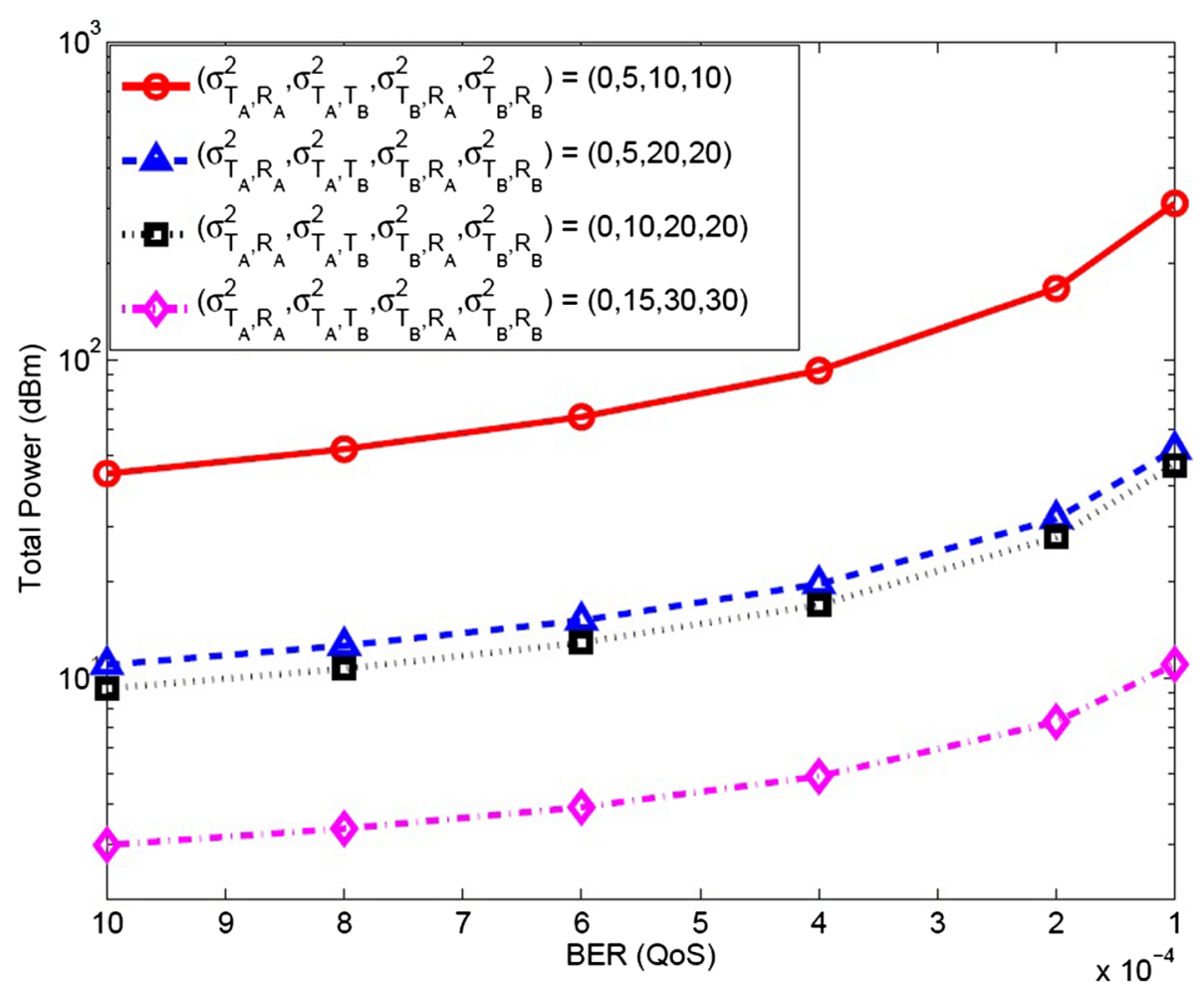

Fig. 6 Variation of total power consumption with QoS requirement given different channel conditions 
link quality of $(0,5,20,20)$ is almost identical to that for a channel link quality of $(0,10,20,20)$. However, for channel link qualities of $(0,5,10,10)$ and $(0,5,20,20)$, a significant difference in the power consumption is observed. These findings are to be expected since, in the former case, only link $T_{A}$-to- $T_{B}$ has a good channel quality. As a result, the power of $U_{A}$ is decreased, while that of $U_{B}$ is potentially increased under the SC scheme. By contrast, in the latter case, both links $T_{B}$-to- $R_{A}$ and $T_{B}$-to- $R_{B}$ have favorable channel quality. Consequently, the powers allocated to $U_{A}$ and $U_{B}$ are both decreased, with the result that the total power consumption is also decreased.

\section{Conclusions}

This paper has considered the problem of optimizing the power allocation strategy in cooperative spectrum-sharing cognitive CR systems. Given the use of a SC scheme at $T_{B}$, analytical expressions have been derived for the corresponding BERs at $R_{A}$ and $R_{B}$. To determine the optimal SC power allocation factor, two more tractable BER expressions have been proposed for $U_{A}$ and $U_{B}$. The allocation problem has been formulated as an optimization problem in which the aim is to minimize the total power consumption of the two users while simultaneously satisfying their BER constraints. Notably, the proposed BER approximations enable the optimization problem to be expressed as a convex formulation amenable to numerical processing. The simulation results have confirmed the ability of the proposed power allocation strategy to achieve the optimal power distribution over a range of realistic CR conditions.

\section{Appendix A: Derivation of $\tilde{\boldsymbol{P}}_{\boldsymbol{e}, \boldsymbol{A}}$}

The second term of (11), denoted as $I_{1}^{R_{A}}$, can be computed as

$$
\begin{aligned}
I_{1}^{R_{A}}= & \int_{0}^{\infty} \int_{0}^{\infty} P_{e, s_{B}}^{R_{A} I}\left(h_{T_{B}, R_{A}}\right) P_{e, s_{A}}^{R_{A}, I I}\left(h_{T_{A}, R_{A}}\right) \\
& \times e^{-\frac{\left|h_{T_{A}, R_{A}}\right|^{2}}{\gamma_{T_{A}, R_{A}}}-\frac{\left.h_{T_{B}, R_{A}}\right|^{2}}{\gamma_{T_{B}, R_{A}}} d\left|h_{T_{A}, R_{A}}\right|^{2} d\left|h_{T_{B}, R_{A}}\right|^{2}} \\
= & \left(\frac{1}{2} \sum_{j=0}^{1} \int_{0}^{\infty} Q\left(\sqrt{\frac{P_{B} \sigma_{T_{B}, R_{A}}^{2}}{\sigma_{n, R_{A}}^{2}}\left(\sqrt{1-\delta}+(-1)^{j} \sqrt{\delta}\right)^{2} x} e^{-x} d x\right)\right. \\
& \times\left(\int_{0}^{\infty} Q\left(\sqrt{2 \frac{P_{T_{B}, R_{A}}(j)}{\sigma_{T_{A}, R_{A}}^{2}} y}\right) e^{-y} d y\right)
\end{aligned}
$$

where $x$ and $y$ are exponentially distributed random variables with unit mean. $\Xi_{T_{B}, R_{A}}(j)$ is further defined as

$$
\Xi_{T_{B}, R_{A}}(j)=\frac{P_{B} \sigma_{T_{B}, R_{A}}^{2}}{\sigma_{n, R_{A}}^{2}}\left(\sqrt{1-\delta}+(-1)^{j} \sqrt{\delta}\right)^{2} .
$$

The Q-function can be expressed as [22]

$$
Q(\sqrt{2 a x})=\frac{1}{\pi} \int_{0}^{\frac{\pi}{2}} e^{-\frac{a x}{\sin ^{2} \theta}} d \theta
$$

(36) can thus be formulated with (38) and its upper bound obtained, i.e., 


$$
\begin{aligned}
I_{1}^{R_{A}} & =\left(\frac{1}{2} \sum_{j=0}^{1} \int_{0}^{\infty} \frac{1}{\pi} \int_{0}^{\frac{\pi}{2}} e^{-\frac{\Xi_{T_{B}, R_{A}(j) x}}{\sin ^{2} \theta}} d \theta e^{-x} d x\right) \int_{0}^{\infty} \frac{1}{\pi} \int_{0}^{\frac{\pi}{2}} e^{-\frac{P_{A} \sigma_{T_{A}, R_{A}}^{2} \sigma_{n, R_{A}}^{2}}{\sin ^{2} \phi}} d \phi e^{-y} d x \\
& =\left(\frac{1}{2} \sum_{j=0} \frac{1}{\pi} \int_{0}^{\frac{\pi}{2}} \frac{\sin ^{2} \theta}{\sin ^{2} \theta+\Xi_{T_{B}, R_{A}}(j)} d \theta\right) \frac{1}{\pi} \int_{0}^{\frac{\pi}{2}} \frac{\sin ^{2} \phi}{\sin ^{2} \phi+\frac{P_{A} \sigma_{T_{A}, R_{A}}^{2}}{\sigma_{n, R_{A}}^{2}} d \phi} \\
\frac{P_{A} \sigma_{T_{A}, R_{A}}^{2}}{\sigma_{n, R_{A}}^{2}} \rightarrow \infty & \left(\frac{1}{2} \sum_{j=0}^{1} \frac{1}{\pi} \int_{0}^{\frac{\pi}{2}} \frac{\sin ^{2} \theta}{\Xi_{T_{B}, R_{A}}(j)} d \theta\right)\left(\frac{1}{\pi} \int_{0}^{\frac{\pi}{2}} \frac{\sin ^{2} \phi}{\frac{P_{A} \sigma_{T_{A}, R_{A}}^{2}}{\sigma_{n, R_{A}}^{2}}} d \phi\right) \\
& =\frac{1}{8}\left(\frac{1}{\Xi_{T_{B}, R_{A}(0)}}+\frac{1}{\Xi_{T_{B}, R_{A}}(1)}\right) \frac{1}{4} \frac{\sigma_{n, R_{A}}^{2}}{P_{A} \sigma_{T_{A}, R_{A}}^{2}} \\
& =\frac{\sigma_{n, R_{A}}^{4}}{32 P_{B} P_{A} \sigma_{T_{A}, R_{A}}^{2} \sigma_{T_{B}, R_{A}}^{2}}\left(\frac{1}{\left(\sqrt{1-\delta}+(-1)^{j} \sqrt{\delta}\right)^{2}}+\frac{1}{\left(\sqrt{1-\delta}+(-1)^{j} \sqrt{\delta}\right)^{2}}\right) \\
& =\frac{\sigma_{n, R_{A}}^{4}}{32 P_{B} P_{A} \sigma_{T_{A}, R_{A}}^{2} \sigma_{T_{B}, R_{A}}^{2}} \frac{2}{(1-2 \delta)^{2}} .
\end{aligned}
$$

The first term, denoted as $I_{2}^{R_{A}}$, can be obtained as

$$
\begin{aligned}
& I_{2}^{R_{A}}=\int_{0}^{\infty} \int_{0}^{\infty} P_{e, s_{A}}^{R_{A},,(M R C)}\left(h_{T_{A}, R_{A}}, h_{T_{B}, R_{A}}\right) e^{-\frac{\left|h_{T_{A}, R_{A}}\right|^{2}}{\gamma_{T_{A}, R_{A}}}-\frac{\left|h_{T_{B}, R_{A}}\right|^{2}}{\gamma_{T_{B}, R_{A}}}} d\left|h_{T_{A}, R_{A}}\right|^{2} d\left|h_{T_{B}, R_{A}}\right|^{2} \\
& -\int_{0}^{\infty} \int_{0}^{\infty} P_{e, s_{B}}^{R_{A}, I}\left(h_{T_{B}, R_{A}}\right) P_{e, s_{A}}^{R_{A}, I(M R C)}\left(h_{T_{A}, R_{A}}, h_{T_{B}, R_{A}}\right) d\left|h_{T_{A}, R_{A}}\right|^{2} d\left|h_{T_{B}, R_{A}}\right|^{2} \\
& =\int_{0}^{\infty} \int_{0}^{\infty} Q\left(\sqrt{2\left(\frac{P_{A} \sigma_{T_{A}, R_{A}}^{2} x}{\sigma_{n, R_{A}}^{2}}+\frac{P_{B} \sigma_{T_{B}, R_{A}}^{2} y \delta}{\sigma_{n, R_{A}}^{2}}\right)}\right) e^{-x-y} d x d y \\
& -\int_{0}^{\infty} \int_{0}^{\infty} \frac{1}{2} \sum_{j=0}^{1} Q\left(\sqrt{2 \Xi_{T_{B}, R_{A}}(j) x}\right) Q\left(\sqrt{2\left(\frac{P_{A} \sigma_{T_{A}, R_{A}}^{2} x}{\sigma_{n, R_{A}}^{2}}+\frac{P_{B} \sigma_{T_{B}, R_{A}}^{2} y \delta}{\sigma_{n, R_{A}}^{2}}\right)}\right) e^{-x-y} d x d y \\
& =\int_{0}^{\infty} \int_{0}^{\infty} \int_{0}^{\infty} \frac{1}{\pi} \int_{0}^{\frac{\pi}{2}} e^{-\frac{P_{A} \sigma_{T_{A}, R_{A}}^{2}{ }_{n, R_{A}}^{2}+\frac{P_{B} \sigma_{T_{B}, R_{A}}^{2} \delta y}{\sigma_{n, R_{A}}^{2}}}{\sin ^{2} \theta}} d \theta e^{-x-y} d x d y \\
& -\int_{0}^{\infty} \int_{0}^{\infty}\left(\frac{1}{\pi} \int_{0}^{\frac{\pi}{2}} \frac{1}{2} \sum_{j=0}^{1} e^{-\frac{\Xi_{T_{B}, R_{A}(j) x}}{\sin ^{2} \theta_{1}}} d \theta_{1}\right) \frac{1}{\pi} \int_{0}^{\frac{\pi}{2}} e^{-\frac{\frac{P_{A} \sigma_{T_{A}, R_{A}{ }^{2}}^{2}+\frac{P_{B} \sigma_{T_{B}, R_{A}}^{2} \delta y}{\sigma_{n, R_{A}}}}{\sin ^{2} \theta_{2}}}{\sigma_{n, R_{A}}}} d \theta_{2} e^{-x-y} d x d y \\
& =\frac{1}{\pi} \int_{0}^{\infty} \int_{0}^{\infty} e^{-\left(\frac{{ }_{A} \sigma_{T_{A}}^{2}, R_{A}}{\sigma_{n, R_{A}}^{2} \sin ^{2} \theta}+1\right) x} d x \int_{0}^{\infty} e^{-\left(\frac{P_{B} \sigma_{T_{B}, R_{A}}^{2}}{\sigma_{n, R_{A}}^{2} \sin ^{2} \theta}+1\right) y} d y d \theta \\
& -\frac{1}{\pi} \frac{1}{\pi} \int_{0}^{\frac{\pi}{2}} \int_{0}^{\frac{\pi}{2}} \int_{0}^{\infty} \frac{1}{2} \sum_{j=0}^{1} e^{-\left(\left(\frac{P_{A} \sigma_{T_{A}, R_{A}}^{2}}{\sin ^{2} \theta_{2} \sigma_{n, R_{A}}^{2}}+\frac{\Xi_{T_{B}, R_{A}(j)}}{\sin ^{2} \theta_{1}}\right)+1\right) x} d x \int_{0}^{\infty} e^{-\left(\frac{P_{B} \sigma_{T_{B}, R_{A} \delta^{2}}^{2}}{\sigma_{n, R_{A}}^{2} \sin ^{2} \theta_{2}}+1\right) y} d y d \theta_{1} d \theta_{2}
\end{aligned}
$$




$$
\begin{aligned}
& =\frac{1}{\pi} \int_{0}^{\frac{\pi}{2}} \frac{\sin ^{2} \theta}{\sin ^{2} \theta+\frac{P_{A} \sigma_{T_{A}, R_{A}}^{2}}{\sigma_{n, R_{A}}^{2}}} \frac{\sin ^{2} \theta}{\sin ^{2} \theta+\frac{\delta P_{B} \sigma_{T_{B}, R_{A}}^{2}}{\sigma_{n, R_{A}}^{2}}} d \theta \\
& -\frac{1}{\pi} \frac{1}{\pi} \int_{0}^{\frac{\pi}{2}} \int_{0}^{\frac{\pi}{2}} \frac{1}{2} \sum_{j=0}^{1} \frac{1}{\left(\frac{P_{A} \sigma_{T_{A}, R_{A}}^{2}}{\sin ^{2} \theta_{2} \sigma_{n, R_{A}}^{2}}+\frac{\Xi_{T_{B}, R_{A}(j)}}{\sin ^{2} \theta_{1}}\right)+1} \frac{1}{\frac{P_{B} \sigma_{T_{B}, R_{A}}^{2} \delta}{\sigma_{n, R_{A}}^{2} \sin ^{2} \theta_{2}}+1} d \theta_{1} d \theta_{2} \\
& \approx \frac{1}{\pi} \int_{0}^{\frac{\pi}{2}} \frac{\sin ^{2} \theta}{\frac{P_{A} \sigma_{T_{A}, R_{A}}^{2}}{\sigma_{n, R_{A}}^{2}}} \frac{\sin ^{2} \theta}{P_{B} \delta \sigma_{T_{B}, R_{A}}^{2}} d \theta=\frac{3}{\sigma_{n, R_{A}}^{2}} \frac{\sigma_{n, R_{A}}^{4}}{P_{A} P_{B} \delta \sigma_{T_{A}, R_{A}}^{2} \sigma_{T_{B}, R_{A}}^{2}} .
\end{aligned}
$$

Note that the approximation given in (45) is followed by ignoring the second term of (44) whenever $\frac{P_{A} \sigma_{T_{A}, R_{A}}^{2}}{\sigma_{n, R_{A}}^{2}}$ or $\frac{P_{B} \sigma_{T_{B}, R_{A}}^{2}}{\sigma_{n, R_{A}}^{2}}$ approaches infinity, i.e.,

$$
\begin{aligned}
& \frac{1}{\left(\frac{P_{A} \sigma_{T_{A}, R_{A}}^{2}}{\sin ^{2} \theta_{2} \sigma_{n, R_{A}}^{2}}+\frac{\Xi_{T_{B}, R_{A}}(j)}{\sin ^{2} \theta_{1}}\right)+1} \frac{1}{\frac{P_{B} \sigma_{T_{B}, R_{A}}^{2} \delta}{\sigma_{n, R_{A}}^{2} \sin ^{2} \theta_{2}}+1} \\
< & \frac{1}{\min \left(\frac{P_{A} \sigma_{T_{A}, R_{A}}^{2}}{\sigma_{n, R_{A}}^{2}}, \Xi_{T_{B}, R_{A}}(j)\right)} \frac{\sin ^{2} \theta_{2}}{\frac{P_{B} \sigma_{T_{B}, R_{A}}^{2} \delta}{\sigma_{n, R_{A}}^{2}}} \rightarrow 0 .
\end{aligned}
$$

Combining (39) and (45) gives

$$
\begin{aligned}
P_{e, s_{A}}^{R_{A}, I}\left(R_{A, S}\right) & \approx I_{1}^{R_{A}}+I_{2}^{R_{A}} \\
& =\frac{\sigma_{n, R_{A}}^{4}}{16 P_{A} P_{B} \sigma_{T_{A}, R_{A}}^{2} \sigma_{T_{B}, R_{A}}^{2}}\left(\frac{1}{(1-2 \delta)^{2}}+\frac{3}{\delta}\right) .
\end{aligned}
$$

Similarly, $P_{e, S_{A}}^{R_{A}, I I}$ can be asymptotically approximated as

$$
\begin{aligned}
P_{e, S_{A}}^{R_{A}, I I} & =\int_{0}^{\infty} Q\left(\sqrt{2\left(\frac{P_{A} \sigma_{T_{A}, R_{A}}^{2} x}{\sigma_{n, R_{A}}^{2}}\right)}\right) e^{-x} d x \\
& =\int_{0}^{\infty} \frac{1}{\pi} \int_{0}^{\frac{\pi}{2}} e^{-\frac{P_{A} \sigma_{T_{A}, R_{A}}^{2}}{\sigma_{n, R_{A}}^{2} \sin ^{2} \theta}} d \theta e^{-x} d x \\
& =\frac{1}{\pi} \int_{0}^{\frac{\pi}{2}} \frac{\sin ^{2} \theta}{\sin ^{2} \theta+\frac{P_{A} \sigma_{T_{A}, R_{A}}^{2}}{\sigma_{n, R_{A}}^{2}}} d \theta \\
& \approx \frac{1}{\pi} \int_{0}^{\frac{\pi}{2}} \frac{\sin ^{2} \theta}{\frac{P_{A} \sigma_{T_{A}, R_{A}}^{2}}{\sigma_{n, R_{A}}^{2}}} d \theta=\frac{\sigma_{n, R_{A}}^{2}}{4 P_{A} \sigma_{T_{A}, R_{A}}^{2}}
\end{aligned}
$$

and $P_{e, s_{A}}^{T_{B}}$ can be also approximated as

$$
\begin{aligned}
P_{e, B_{A}}^{T_{B}} & =\frac{1}{\pi} \int_{0}^{\frac{\pi}{2}} \frac{\sin ^{2} \phi}{\sin ^{2} \phi+\frac{P_{A} \sigma_{T_{A}, T_{B}}^{2}}{\sigma_{n, T_{B}}^{2}}} d \phi \\
& \approx \frac{1}{\pi} \int_{0}^{\frac{\pi}{2}} \frac{\sin ^{2} \phi}{\frac{\sigma_{A} \sigma_{T_{A}, T_{B}}^{2}}{\sigma_{n, T_{B}}^{2}}} d \phi=\frac{\sigma_{n, T_{B}}^{2}}{4 P_{A} \sigma_{T_{A}, T_{B}}^{2}} .
\end{aligned}
$$


Finally, substituting (47), (48), and (49) into (11), we have

$$
\begin{aligned}
P_{e, A}= & \left(1-P_{e, s_{A}}^{T_{B}}\right) P_{e, s_{A}}^{R_{A}, I I}+P_{e, s_{A}}^{T_{B}} P_{e, s_{A}}^{R_{A}, I} \\
\approx & \frac{\sigma_{n, T_{B}}^{2}}{4 P_{A} \sigma_{T_{A}, T_{B}}^{2}} \frac{\sigma_{n, R_{A}}^{2}}{4 P_{A} \sigma_{T_{A}, R_{A}}^{2}} \\
& +\left(1-\frac{1}{4} \frac{\sigma_{n, T_{B}}^{2}}{P_{A} \sigma_{T_{A}, T_{B}}^{2}}\right) \frac{\sigma_{n, R_{A}}^{4}}{16 P_{A} P_{B} \sigma_{T_{A}, R_{A}}^{2} \sigma_{T_{B}, R_{A}}^{2}}\left(\frac{1}{(1-2 \delta)^{2}}+\frac{3}{\delta}\right)
\end{aligned}
$$

which completes the proof of $\widetilde{P}_{e, A}$.

\section{Appendix B: Derivation of $\tilde{\boldsymbol{P}}_{\boldsymbol{e}, \boldsymbol{B}}$}

Similarly, $P_{e, s_{B}}^{R_{B}, I I}$ can be asymptotically approximated using the derivation in (48) as

$$
\begin{aligned}
P_{e, s_{B}}^{R_{B}, I I} & =\frac{1}{\pi} \int_{0}^{\frac{\pi}{2}} \frac{\sin ^{2} \phi}{\sin ^{2} \phi+\frac{P_{B} \sigma_{T_{B}, R_{B}}^{2}}{\sigma_{n, R_{B}}^{2}}} d \phi \\
& \approx \frac{1}{\pi} \int_{0}^{\frac{\pi}{2}} \frac{\sin ^{2} \phi}{\frac{\sigma_{n, R_{B}}^{2}}{P_{B} \sigma_{T_{B}, R_{B}}^{2}}} d \phi=\frac{\sigma_{n, R_{B}}^{2}}{4 P_{B} \sigma_{T_{B}, R_{B}}^{2}} .
\end{aligned}
$$

The approximated $P_{e, s_{B}}^{R_{B}, I}$ can be computed as

$$
\begin{aligned}
P_{e, s_{B}}^{R_{B}, I} & =\int_{0}^{\infty} \frac{1}{2} \sum_{i=0}^{1} Q\left(\sqrt{2 \frac{P_{B}\left|h_{T_{B}, R_{B}}\right|^{2}}{\sigma_{n, R_{B}}^{2}}\left(\sqrt{1-\delta}+(-1)^{j} \sqrt{\delta}\right)^{2}}\right) e^{-\frac{\left.h_{T_{B}, R_{B}}\right|^{2}}{\gamma_{T_{B}, R_{B}}}} d\left|h_{T_{B}, R_{B}}\right|^{2} \\
& =\frac{1}{2}\left[\sum_{i=0}^{1} \int_{0}^{\infty} Q\left(\sqrt{2 \frac{P_{B}\left|h_{T_{B}, R_{B}}\right|^{2}}{\sigma_{n, R_{B}}^{2}}\left(\sqrt{1-\delta}+(-1)^{j} \sqrt{\delta}\right)^{2}}\right) e^{-\frac{\left|h_{T_{B}, R_{B}}\right|^{2}}{\gamma_{T_{B}, R_{B}}}} d \mid h_{T_{B},\left.R_{B}\right|^{2}}\right] \\
& \approx \frac{1}{2}\left[\sum_{i=0}^{1} \frac{\sigma_{n, R_{B}}^{2}}{4 P_{B} \sigma_{T_{B}, R_{B}}^{2}} \frac{1}{\left(\sqrt{1-\delta}+(-1)^{j} \sqrt{\delta}\right)^{2}}\right] \\
& =\frac{1}{8} \frac{\sigma_{n, R_{B}}^{2}}{P_{B} \sigma_{T_{B}, R_{B}}^{2}}\left[\frac{1}{(\sqrt{1-\delta}+\sqrt{\delta})^{2}}+\frac{1}{(\sqrt{1-\delta}-\sqrt{\delta})^{2}}\right] \\
& =\frac{\sigma_{n, R_{B}}^{2}}{8 \sigma_{T_{B}, R_{B}}^{2} P_{B}} \frac{2}{(1-2 \delta)^{2}} \cdot
\end{aligned}
$$

Finally, substituting (49), (51), and (52) into (15) gives

$$
\begin{aligned}
P_{e, B}= & \left(1-P_{e, s_{A}}^{T_{B}}\right) P_{e, s_{B}}^{R_{B}, I}+P_{e, s_{A}}^{T_{B}} P_{e, s_{B}}^{R_{B}, I I} \\
\approx & \left(\frac{\sigma_{n, T_{B}}^{2}}{4 \sigma_{T_{A}, T_{B}}^{2} P_{A}}\right) \times\left(\frac{\sigma_{n, R_{B}}^{2}}{4 \sigma_{T_{B}, R_{B}}^{2} P_{B}}\right) \\
& +\left(1-\frac{\sigma_{n, T_{B}}^{2}}{4 \sigma_{T_{A}, T_{B}}^{2} P_{A}}\right) \times\left(\frac{\sigma_{n, R_{B}}^{2}}{8 \sigma_{T_{B}, R_{B}}^{2} P_{B}} \frac{2}{(1-2 \delta)^{2}}\right)
\end{aligned}
$$

which completes the derivation of $\widetilde{P}_{e, B}$. 


\section{Endnotes}

${ }^{1}$ It is noteworthy that dirty paper coding (DPC) may be applied at $T_{B}$ to convey $s_{A}$ and $s_{B}$ which is theoretical optimal in a 2-user SISO Gaussian broadcasting channel system [25]. However, DPC relies on the perfect channel state information to achieve optimal performance and is vulnerable to channel estimation error. Thus, DPC is not considered herein for it violating our economic scenario where only the statistical channel variance is required.

${ }^{2}$ If $s_{B}$ is not detected in advance, a MRC operation can not optimally be adopted to facilitate the design of power allocation.

\section{Abbreviations}

BER: Bit error rate; CR: Cognitive radio; DF: Decode-and-forward; DPC: Dirty paper coding; MRC: Maximum ratio combining; QoS: Quality-of-service; SC: Superposition coding.

\section{Author details}

${ }^{1}$ Department of Communications Engineering, Feng Chia University, Taichung,

40724 Taiwan. ${ }^{2}$ Institute of Communications Engineering, National Sun

Yat-sen University, Building F, No. 70, Lienhai Rd., Kaohsiung, 80424 Taiwan.

Received: 16 June 2015 Accepted: 6 October 2015

Published online: 24 November 2015

\section{References}

1. J Mitola, Dissertation, KTH (2000)

2. S Haykin, Cognitive radio: brain-empowered wireless communications. IEEE J. Sel. Areas. Commun. 23, 201-220 (2005)

3. A Goldsmith, S Jafar, I Maric, S Srinivasa, Breaking spectrum gridlock with cognitive radios: an information theoretic perspective. Proc. of the IEEE. 97, 894-914 (2009)

4. R Prasad, P Pawelczak, J Hoffmeyer, H Berger, Cognitive functionality in next generation wireless networks: standardization efforts. IEEE Commun. Mag. 46, 72-78 (2008)

5. R Blasco-Serrano, J LV, R Thobaben, E Jorswieck, A Kliks, M Skoglund, in Proceedings of IEEE CROWNCOM. Comparison of underlay and overlay spectrum sharing trategies in MISO cognitive channels, (2012)

6. R Manna, R Louie, Y Li, B Vucetic, Cooperative spectrum sharing in cognitive radio networks with multiple antennas. IEEE Trans. Signal Process. 59, 5509-5522 (2011)

7. $V$ Bohara, S Ting, Y Han, A Pandharipande, in Proceedings of IEEE CROWNCOM. Interference-free overlay cognitive radio network based on cooperative space time coding, (2012)

8. N Devroye, P Mitran, $\mathrm{V}$ Tarokh, Achievable rates in cognitive radio channels. IEEE Trans. Inf. Theory. 52, 1813-1827 (2006)

9. A Jovicic, P Viswanath, Cognitive radio: an information-theoretic perspective. IEEE Trans. Inf. 55, 3945-3958 (2009)

10. R Zhang, Y-C Liang, Exploiting multi-antennas for opportunistic spectrum sharing in cognitive radio networks. IEEE J. Top. Signal Process. 2, 88-102 (2008)

11. J Ma, G Zhao, Y Li, Soft combination and detection for cooperative spectrum sensing in cognitive radio networks. IEEE Trans. Wirel. Commun. 7, 4502-4507 (2008)

12. $Y$ Zou, Y-D Yao, B Zheng, A cooperative sensing based cognitive relay transmission scheme without a dedicated sensing relay channel in cognitive radio network. IEEE Trans. Signal Process. 59, 854-858 (2011)

13. R Blasco-Serrano, J LV, R Thobaben, E Jorswieck, M Skoglund, Multi-antenna transmission for underlay and overlay cognitive radio with explicit message learning phase. EURASIP J. Wirel. Commun. Netw. 7, 1-21 (2013). doi:1687-1499-2013-195

14. N Zhao, H Sun, Robust power control for cognitive radio in spectrum underlay networks. KSII Trans. Internet and Inf. Syst. 7, 1214-1229 (2011)
15. N Zhao, F Yu, H Sun, M Li, Adaptive power allocation schemes for spectrum sharing in interference alignment (IA)-based cognitive radio network. IEEE Trans Veh. Technol, 1-14 (2016). doi:10.1109/TVT.2015.2440428

16. C-S Ricardo, L Jing, T Ragna, J Eduard, K Adrian, S Mikael, in Proceedings of IEEE CROWNCOM. Comparison of underlay and overlay spectrum sharing strategies in MISO cognitive channels, (2012)

17. P Popoviski, E Carvalho, Improving the rates in wireless relay systems through superposition coding. IEEE Trans. Wireless Commun. 7, 4831-4836 (2008)

18. F-S Tseng, C-Y Hsu, Robust multiple antenna cooperative spectrum sharing design with random vector quantization. IEEE Trans. Veh. Technol. 63, 3232-3249 (2014)

19. Y Han, A Pandharipande, S Ting, Cooperative decode-and-forward relaying for secondary spectrum access. IEEE Trans. Wireless Commun. 8 4945-4950 (2009)

20. I Maric, R Yates, G Kramer, Capacity of interference channels with partial transmitter cooperation. IEEE Trans. Inf. Theory. 53, 3536-3548 (2007)

21. O Simeone, I Stanojev, S Savazzi, Y Bar-Ness, U Spagnolini, R Pickholtz, Spectrum leasing to cooperating secondary and hoc networks. IEEE J. Selected Areas in Commun. 26, 203-213 (2008)

22. M Simon, M Alouini, Digitial Communication over Fading Channels - $A$ Unified Approach to Performance Analysis. (Wiley-Interscience, New York, 2000)

23. G Cardano, ARS MAGNA or The Rules of Algebra (Dover, USA,1968)

24. http://cvxr.com/cvx/. Accessed 2 October 2015

25. S Yang, J Belfiore, in Proceedings of the 43rd Allerton Conference. The impact of channel estimation error on the DPC region of the two-user Gaussian broadcast channel (Monticello, IL, USA, 2005)

\section{Submit your manuscript to a SpringerOpen ${ }^{\circ}$ journal and benefit from:}

- Convenient online submission

Rigorous peer review

- Immediate publication on acceptance

- Open access: articles freely available online

- High visibility within the field

- Retaining the copyright to your article

Submit your next manuscript at $>$ springeropen.com 